\title{
Robustness Of Stochastic Learning Dynamics To Player Heterogeneity In Games
}

\author{
Hassan Jaleel, Waseem Abbas, and Jeff S. Shamma .
}

\begin{abstract}
We investigate the impact of players with heterogeneous update rules on the long-term behavior of a population under stochastic learning dynamics. We show that under certain conditions, the presence of even a single heterogeneous player with a different decision making strategy can significantly alter the long-term behavior of the entire population. To quantify the impact of a heterogeneous player, we define a new notion of robustness of stochastic learning dynamics to player heterogeneity. Based on our proposed notion, an action profile that is stochastically stable under the standard setup is robust to player heterogeneity if it can still explain the long-run behavior of all the players other than the heterogeneous players. We consider two types of heterogeneous players: A confused player who randomly updates his actions and a stubborn player who never updates his action. For each of these types, we present a qualitative description of scenarios in which an action profile that is stochastically stable under the standard setup is not robust to the presence of a heterogeneous player of a particular type.
\end{abstract}

\section{INTRODUCTION}

In the theory of learning in games, the objective is to understand the interactions among rational decision makers and how such interactions may or may not lead to the emergence of an equilibrium behavior in the long run. Stochastic learning dynamics is a class of learning dynamics in which agents are assumed to have bounded rationality. The standard setup in these dynamics comprises a large population of players in which each player updates his action according to some version of noisy best/better response to the actions of other players. Some important examples of stochastic learning dynamics are Log-Linear Learning (LLL), Adaptive play, and Metropolis learning (see e.g., [1], [2], [3], [4], [5], and [6] and the references therein).

A fundamental assumption in the existing stochastic learning dynamics is that the players are homogeneous, i.e., all the players have the same strategy for updating their actions. In practical scenarios particularly involving large populations of independent decision makers, this assumption may be overly restrictive. Even though bounded rationality enables us to model a certain level of noise in decision making, there can exist players whose update strategy cannot be approximated by noisy best/better response dynamics. Such

H. Jaleel is with the Department of Electrical Engineering, Syed Babar Ali School of Science \& Engineering at Lahore University of Management Science (LUMS), Lahore, Pakistan. W. Abbas is with the Department of Electrical Engineering and Computer Science at Vanderbilt University, Nashville, TN 37212, USA. J.S. Shamma is with the Robotics, Intelligent Systems \& Control (RISC) Lab, Computer, Electrical and Mathematical Sciences and Engineering Division (CEMSE) at King Abdullah University of Science and Technology (KAUST), Thuwal 23955-6900, Saudi Arabia. Emails: hassan.jaleel@lums.edu.pk, waseem.ee@gamil.com, jeff.shamma@kaust.edu.sa players introduce heterogeneity of decision making rules, which is not considered in the standard setup.

Analyzing the long-term behavior of stochastic learning dynamics has been an active area of research for a long time. However, stochastic learning dynamics with heterogeneous players has not received significant research attention. We argue that the analysis of stochastic learning dynamics in games with heterogeneous players requires serious research attention by showing that the presence of even a single heterogeneous player can significantly alter the long-term behavior of a large population of players under certain conditions. Recent literature on network dynamics with heterogeneous players like [7], [8], [9], and [10] is closely related to the problem under investigation. However, the focus of this literature has been on opinion and consensus dynamics in the presence of heterogeneous players. We propose a formal analysis framework to analyze the possible impacts of player heterogeneity on the long-run behavior of a population of players under a general class of stochastic learning dynamics in games.

To quantify the impact of heterogeneous players, our primary contribution is to introduce a new notion of robustness to player heterogeneity. In particular, we consider two types of heterogeneous players: a confused player and a stubborn player. In our model, given the opportunity to update their actions, a confused player updates his action uniformly at random and a stubborn player never updates his action. For each of these heterogeneous player types, we analyze the robustness of stochastic learning dynamics based on our proposed notion of robustness to heterogeneity.

Starting from the standard setup, we assume that a single player, say player $h$, is replaced with a heterogeneous player of a particular type. We define that an action profile that was stochastically stable under the standard setup is robust to player heterogeneity of that type with respect to player $h$ if the replacement of player $h$ with a heterogeneous player cannot impact the long-run behavior of the rest of the population. Thus, if an action profile is stochastically stable under the standard setup, it is still a valid description of the long-run behavior of the population even if player $h$ is replaced with a heterogeneous player. A stochastically stable action profile is robust to player heterogeneity of a particular type if it is robust with respect to all the players. Finally, a stochastic learning dynamics is robust to player heterogeneity if all the stochastically stable action profiles under the standard setup are robust to player heterogeneity.

After defining robustness of stochastic learning dynamics to player heterogeneity, we analyze the impact of having 
a confused player or a stubborn player in the population. We derive conditions in which a stochastically stable action profile under the standard setup will not be robust to player heterogeneity of a particular type. In the case of a confused player, we also analyze conditions in which an action profile that was not stochastically stable under the standard setup will become stochastically stable under the heterogeneous setup. Our robustness analysis is based on the radius and coradius based analysis that was originally presented in [11], and later generalized in [4] for a class of stochastic learning dynamics.

\section{BACKGROUND}

We denote the cardinality of a set $S$ by $|S|$. The distance between two vectors $x$ and $y$ in $\mathbb{R}^{n}$ is

$$
d_{H}(x, y)=\left|\left\{j \mid x_{j} \neq y_{j}\right\}\right| .
$$

Here $d_{H}(x, y)$ is the Hamming distance between $x$ and $y$.

Let $X_{0}$ be an unperturbed Markov chain defined over a state space $S$ and let $P_{0}$ be the transition matrix for $X_{0}$. Suppose that $X_{0}$ is perturbed and let $X_{\epsilon}$ be a family of perturbed Markov chains with transition matrix $P_{\epsilon}$ where $\epsilon$ is a sufficiently small number. The perturbed chain $X_{\epsilon}$ is called a regular perturbation of $X_{0}$ if it satisfies the following properties:

- $P_{\epsilon}$ is ergodic when $\epsilon$ is sufficiently small,

- $\lim _{\epsilon \rightarrow 0} P_{\epsilon}(x, y)=P_{0}(x, y)$ for any state pair $x$ and $y$ in $\stackrel{\epsilon,}{\text { and }}$

- There exists a function $R(x, y)$ such that if $P_{\epsilon}(x, y)>0$ for some $\epsilon>0$ then

$$
0<\lim _{\epsilon \rightarrow 0} \frac{P_{\epsilon}(x, y)}{\epsilon^{R(x, y)}}<\infty .
$$

Here $R(x, y)$ is called the resistance in transition from $x$ to $y$.

Let $\pi_{\epsilon}$ be the unique stationary distribution for a regularly perturbed Markov chain $X_{\epsilon}$ that is ergodic and reversible. A state $x \in S$ is stochastically stable for $X_{\epsilon}$ if

$$
\lim _{\epsilon \rightarrow 0} \pi_{\epsilon}(x)>0 \text {. }
$$

Thus, a state is stochastically stable if it has a non-zero probability in the stationary distribution in the limit as $\epsilon \rightarrow 0$.

For any state pair $x$ and $y$ in $S$, a path $\omega_{x, y}$ is a sequence of states $\left(\omega_{0}, \omega_{1}, \ldots, \omega_{k}\right)$ such that $\omega_{0}=x, \omega_{k}=y, \omega_{i} \in$ $S, \omega_{i} \neq \omega_{j}$ for any $i$ and $j$, and $P\left(\omega_{i}, \omega_{i+1}\right)>0$ for all $i \in\{0,1, \ldots, k-1\}$. We denote the length of the path by $\left|\omega_{x, y}\right|$. We say that $\omega_{x, y} \in A$ for any $A \subset S$ if $\omega_{i} \in A$ for all $i \in\{0,1, \ldots, k\}$. Let $\Omega(x, y)$ be the set of all the paths between the states $x$ and $y$. State $y$ is reachable from state $x(x \rightarrow y)$ if $\Omega(x, y)$ is nonempty. Moreover, states $x$ and $y$ can communicate with each other $(x \leftrightarrow y)$ if $\Omega(x, y)$ and $\Omega(y, x)$ are both nonempty. For any two subsets $A$ and $B$ of $S$, we define $\Omega(A, B)$ as the set of all paths from states in $A$ to some state in $B$

$$
\Omega(A, B)=\bigcup_{x \in A, y \in B} \Omega(x, y) .
$$

A set $A$ is connected if $x \leftrightarrow y$ for all $x$ and $y$ in $A$.

The resistance of a path $\omega=\left(\omega_{0}, \omega_{1}, \ldots, \omega_{k}\right)$ is

$$
R(\omega)=\sum_{i=0}^{k-1} R\left(\omega_{i}, \omega_{i+1}\right) .
$$

For a state pair $(x, y)$ such that $y \notin \mathcal{N}(x)$, the resistance from $x$ to $y$ is

$$
R^{\Omega}(x, y)=\min _{\omega \in \Omega(x, y)} R(\omega)
$$

\section{A. Game Formulation}

Let $N_{p}=\{1,2, \ldots, n\}$ be the set of players, $A_{i}=$ $\left\{1,2, \ldots, m_{i}\right\}$ be the set of actions of player $i$, and $\mathcal{A}=$ $A_{1} \times A_{2} \times \cdots \times A_{n}$ be the set of joint action profiles. We will represent an action profile a $\in \mathcal{A}$ as $\left(a_{i}, \mathrm{a}_{-i}\right)$, where $a_{i}$ is the action of player $i$ and $\mathrm{a}_{-i}$ is the actions of all the players other than $i$. The preferences of player $i$ over his actions is given in terms of its utility function $U_{i}: \mathcal{A} \rightarrow \mathbb{R}$ where player $i$ prefers action $a_{i}$ over $a_{i}^{\prime}$ if $U_{i}\left(a_{i}, \mathrm{a}_{-i}\right)>U_{i}\left(a_{i}^{\prime}, \mathrm{a}_{-i}\right)$. The best response set of player $i$ to $\mathrm{a}_{-i}$ is

$B_{i}\left(\mathrm{a}_{-i}\right)=\left\{a_{i} \mid U_{i}\left(a_{i}, \mathrm{a}_{-i}\right) \geq U_{i}\left(\mathrm{a}_{i}^{\prime}, \mathrm{a}_{-i}\right)\right.$ for all $\left.a_{i}^{\prime} \in A_{i}\right\}$.

An action profile $\mathrm{a}^{*}$ is a Nash Equilibrium (NE) if and only if $a_{i}^{*} \in B_{i}\left(\mathrm{a}_{-i}^{*}\right)$ for every player $i$, i.e., each player is playing a best response to the actions of other players. We define the neighborhood of an action profile a as follows:

$$
\mathcal{N}(\mathrm{a})=\left\{\mathrm{a}^{\prime} \in \mathcal{A} \mid d_{H}\left(\mathrm{a}, \mathrm{a}^{\prime}\right) \leq 1\right\} .
$$

The neighborhood of a with respect to player $i$ is

$$
\mathcal{N}(\mathrm{a}, i)=\left\{\mathrm{a}^{\prime} \in \mathcal{A} \mid a_{i}^{\prime} \in A_{i} \text { and } \mathrm{a}_{-i}^{\prime}=\mathrm{a}_{-i}\right\} .
$$

A game is an exact potential game if there exists a potential function $\phi: \mathcal{A} \rightarrow \mathbb{R}$ such that

$$
U_{i}\left(a_{i}, \mathrm{a}_{-i}\right)-U_{i}\left(a_{i}^{\prime}, \mathrm{a}_{-i}\right)=\phi\left(a_{i}, \mathrm{a}_{-i}\right)-\phi\left(a_{i}^{\prime}, \mathrm{a}_{-i}\right) .
$$

Let $\omega=\left(\omega_{0}, \omega_{1}, \ldots, \omega_{k}\right)$ be a path from action profile a to $\mathrm{a}^{\prime}$. We define the resistance contribution of player $h$ in $\omega$ as

$$
R(\omega, h)=\sum_{j \in I_{h}(\omega)} R\left(\omega_{j}, \omega_{j+1}\right),
$$

where

$$
I_{h}(\omega)=\left\{j \mid \omega_{j}=\left(a_{h}, \mathrm{a}_{-h}\right) \text { and } \omega_{j+1}=\left(a_{h}^{\prime}, \mathrm{a}_{-h}\right)\right\} .
$$

Thus, $I_{h}(\omega)$ is a set of the indices in the path $\omega$ at which player $h$ updates his action.

\section{B. Stochastic Learning Dynamics}

Stochastic learning dynamics is a class of learning dynamics in which at each time step, players play best or better response to the actions of other players with a high probability. However, with a low but non-zero probability, the players explore the action space by playing an action that may not improve their utility. In this work, we will consider Log-Linear Learning (LLL) as a representative of stochastic 
learning dynamics (see [3] and [6] for details). The resistance between two action profiles a and a' under LLL is

$$
R^{\mathrm{LLL}}\left(\mathrm{a}, \mathrm{a}^{\prime}\right)=\left\{\begin{array}{cl}
U_{i}\left(a_{i}^{*}, \mathrm{a}_{-i}\right)-U_{i}\left(\mathrm{a}^{\prime}\right) & a_{i}^{\prime} \neq a_{i}, \mathrm{a}_{-i}^{\prime}=\mathrm{a}_{-i} \\
\infty & \text { Otherwise }
\end{array}\right.
$$

where $\mathrm{a}_{i}^{*} \in B_{i}\left(\mathrm{a}_{-i}\right)$. It is well established (see e.g. [4]) that for a potential game, $X^{\mathrm{LLL}}$ is ergodic and reversible with Gibbs distribution as stationary distribution, i.e.,

$$
\pi^{\mathrm{LLL}}(\mathrm{a})=\frac{1}{Z} e^{\frac{1}{T} \phi(\mathrm{a})}, \text { where } Z=\sum_{\mathrm{y} \in \mathcal{A}} e^{\frac{1}{T} \phi(\mathrm{y})} .
$$

is the normalizing constant and $T$ is the noise parameter.

1) Radius-Coradius based analysis of stochastic learning dynamics: The radius-coradius based analysis was originally presented in [11] for learning dynamics with mistake model. In the mistake model, the players play best response to the actions of others with a high probability $1-\epsilon$. However, with a small probability $\epsilon$, the updating player makes a mistake typically by choosing an action uniformly at random from his action set. In [4], the radius-coradius analysis was extended to a class of learning dynamics in which magnitude of mistakes (loss of utility) was also considered. Our analysis in this work will be based on the results of [4]. We start by defining three fundamental terms required for this analysis from [4].

Definition 2.1: Let $X$ be a Markov chain defined over the set $S$.

- The basin of attraction of a state $x$ is $\operatorname{BA}(x)$, where

$$
\mathrm{BA}(x)=\{y \in S \mid R(\omega)=0 \text { for some } \omega \in \Omega(y, x)\} \text {. }
$$

The complement of $\mathrm{BA}(x)$ is $\mathrm{BA}^{c}(x)$, i.e., $\operatorname{BA}^{c}(x)=$ $S \backslash \operatorname{BA}(x)$.

- The recurrent class of a state $x$ is $L(x)$ where

$$
L(x)=\{y \in S \mid y \in \operatorname{BA}(x) \text { and } x \in \operatorname{BA}(y)\} .
$$

- The radius of a state $x$ is $R_{d}(x)$ and is defined as follows:

$$
R_{d}(x)=\min \left\{R^{\Omega}(x, y) \mid y \in \mathrm{BA}^{c}(x)\right\} .
$$

- The coradius of a state $x$ is $\mathrm{CR}(x)$ and is defined as follows:

$$
\mathrm{CR}(x)=\max \left\{R^{\Omega}(y, x) \mid y \in \mathrm{BA}^{c}(x)\right\} .
$$

Thus, the radius of a state represents how easy it is to leave that state, and the coradius of a state represents how difficult it is to reach that state starting from any other state in $S$. The recurrent class of state $x$ is the set of all states $y$ such that $x \leftrightarrow y$ and $R^{\Omega}(x, y)=R^{\Omega}(y, x)=0$. For a set $A \subset S$ such that there exist zero resistance paths between any two states $x$ and $y$ in $A$, the radius and coradius can be defined as follows:

$$
\begin{aligned}
R_{d}(A) & =\min \left\{R_{d}(x) \text { for all } x \in A\right\}, \text { and } \\
\mathrm{CR}(A) & =\min \{\mathrm{CR}(x) \text { for all } x \in A\} .
\end{aligned}
$$

Using the concepts of radius and coradius, stochastic stability is defined in Prop. 4 in [4] as follows:
Definition 2.2: Let $x$ be a state that satisfies $R_{d}(x)>$ $\mathrm{CR}(x)$. Then, all the states in $L(x)$ are stochastically stable.

\section{Robustness To Player Heterogeneity}

An important assumption in the standard setup for stochastic learning dynamics is that all the players update their actions based on some version of noisy better or best response. We consider the scenario in which one of the players updates his actions based on an update rule that is different from the update rule employed by the rest of the players. Having a heterogeneous player in the population raises several interesting questions regarding the long-run behavior of the population. In particular, can a single heterogeneous player affect the long-run behavior of the entire population? Are the existing techniques for analyzing stochastic learning dynamics still feasible in the case of a population with heterogeneous players? Most importantly, if the presence of a heterogeneous player can impact the long-run behavior, how to quantify and analyze its impact under stochastic learning dynamics?

To investigate the impact of player heterogeneity on the long-run behavior, we consider two types of heterogeneous players.

- Confused player: Randomly updates his actions.

- Stubborn player: Never updates his action.

For each of the above types of heterogeneous players, we establish that under certain conditions even a single heterogeneous player can significantly alter the long-run behavior of the entire population under a stochastic learning dynamics. To analyze the impact of a heterogeneous player on the longrun behavior of the population, our first contribution is a novel notion of robustness of stochastic learning dynamics to player heterogeneity.

Let $\mathcal{A}_{\mathrm{ss}} \subset \mathcal{A}$ be the set of stochastically stable states under a stochastic learning dynamics if there is no heterogeneous player. We will refer to the setup without a heterogeneous player and with a heterogeneous player as a standard setup and a heterogeneous setup respectively. In the heterogeneous setup, we assume that one of the players from the standard setup is replaced with a heterogeneous player. The heterogeneous player will either be a confused player or a stubborn player. Next, we present our notion of robustness of stochastic learning dynamics to player heterogeneity. In this regard, we define three levels of robustness.

Definition 3.1: Let $\mathcal{A}_{\mathrm{ss}}$ be the set of all stochastically stable action profiles for a stochastic learning dynamics under the standard setup and let $\mathrm{s} \in \mathcal{A}_{\mathrm{ss}}$.

- Let player $h$ be replaced with a heterogeneous player of a particular type and let $\mathcal{A}_{\mathrm{ss}}^{h}$ be the corresponding set of stochastically stable action profiles under the heterogeneous setup. The action profile $\mathrm{s}=\left(s_{h}, \mathrm{~s}_{-h}\right)$ is robust to the replacement of $h$ with a particular type of a heterogeneous player if there exists an $\mathrm{s}^{\prime} \in \mathcal{A}_{\mathrm{ss}}^{h}$ such that $\mathrm{s}_{-h}^{\prime}=\mathrm{s}_{-h}$.

- The action profile $\mathrm{s}$ is robust to player heterogeneity of a particular type if it is robust to the replacement of 
any player with the heterogeneous player of that type under the learning dynamics.

- The learning dynamics is robust to player heterogeneity of a particular type if all $\mathrm{s} \in \mathcal{A}_{\mathrm{ss}}$ are robust to player heterogeneity of that type.

Thus, a stochastically stable action profile under the standard setup is robust with respect to a type of heterogeneous player if replacing any player $h$ with the heterogeneous player cannot affect the behavior of other players in the population. To analyze the impact of a heterogeneous player on the longrun behavior, we thoroughly investigate the two types of heterogeneous players.

\section{Confused Player: Random Action Updates}

We begin with the case in which the heterogeneous player $h$ is a confused player and he randomly updates his actions whenever he gets the opportunity. To keep the analysis simple, we assume that for all $\mathrm{s} \in \mathcal{A}_{\mathrm{ss}}$, the equivalence class $L(\mathrm{~s})$ is empty. The main results related to the addition of a confused player are as follows:

1) Under certain conditions, $\mathrm{s} \in \mathcal{A}_{\mathrm{ss}}$ is not robust to the addition of a confused player.

2) It is possible that $\mathrm{s}$ is robust if some player $h$ is replaced with a confused player but is not robust if some other player $h^{\prime}$ is replaced with a confused player.

3) It is also possible that an action profile a that was not stochastically stable under the standard setup may become stochastically stable after replacing a player with a confused player.

Proposition 1: Let $\mathrm{s} \in \mathcal{A}_{\mathrm{ss}}$ be a stochastically stable action profile in the standard case. Then, $\mathrm{s}$ is robust to the replacement of player $h$ with a confused player if

$$
R_{d}\left(L^{\mathrm{cnf}}(\mathrm{s}, h)\right)>\mathrm{CR}\left(L^{\mathrm{cnf}}(\mathrm{s}, h)\right),
$$

where

$$
L^{\mathrm{cnf}}(\mathrm{s}, h)=\bigcup_{\mathrm{s}^{\prime} \in \mathcal{N}(\mathrm{s}, h)} \mathrm{s}^{\prime},
$$

$\mathcal{N}(\mathrm{s}, h)$ is the player specific neighborhood of $\mathrm{s}$ defined in $(2)$, and $R_{d}(\mathrm{~s}, h)$ and $\mathrm{CR}(\mathrm{s}, h)$ are radius and coradius defined in (5) and (6).

Proof: Action profile s is not robust to the addition of a confused player $h$ if $\mathrm{s}_{-h}$ does not comprise a stochastically stable action profile for some action $a_{h}$ of the heterogeneous player. Since a confused player randomly updates his actions, all the transitions that involve the confused player $h$ have zero resistance, i.e.,

$$
R^{\mathrm{cnf}}\left(\mathrm{a}, \mathrm{a}^{\prime}\right)= \begin{cases}0 & \mathrm{a}^{\prime} \in \mathcal{N}(\mathrm{a}, h) \\ R\left(\mathrm{a}, \mathrm{a}^{\prime}\right) & \mathrm{a}^{\prime} \notin \mathcal{N}(\mathrm{a}, h) .\end{cases}
$$

Since $\mathrm{s}$ is stochastically stable under the standard setup, we know from Def. 2.2 that $R_{d}(\mathrm{~s})>\mathrm{CR}(\mathrm{s})$. However, by replacing $h$ with a confused player, any transition from $\mathrm{s}$ to any member of the set $\mathcal{N}(\mathrm{s}, h)$ has zero resistance, which

\begin{tabular}{c|c|c|c|}
\multicolumn{4}{c}{$U_{c}\left(c_{1}\right)=10$} \\
\multicolumn{1}{c}{$b_{1}$} & $b_{2}$ & \multicolumn{1}{c}{$b_{3}$} \\
\cline { 2 - 4 }$a_{1}$ & 10 & 6 & 7 \\
\cline { 2 - 4 }$a_{2}$ & 6 & 0 & 0 \\
\cline { 2 - 4 }$a_{3}$ & 0 & 0 & 9 \\
\cline { 2 - 4 } & & &
\end{tabular}

\begin{tabular}{c|c|c|c|}
\multicolumn{4}{c}{$U_{c}\left(c_{1}\right)=5$} \\
\multicolumn{1}{c}{$b_{1}$} & $b_{2}$ & $b_{3}$ \\
\cline { 2 - 4 }$a_{1}$ & 10 & 0 & 0 \\
\cline { 2 - 4 }$a_{2}$ & 0 & 3 & 3 \\
\cline { 2 - 4 }$a_{3}$ & 0 & 3 & 3 \\
\cline { 2 - 4 } & & &
\end{tabular}

Fig. 1. A game with three players $\{a, b, c\}$. Players $a$ and $b$ select the rows and columns of the matrices and player $c$ selects between left and right matrix. Players $a$ and $b$ are identical interest with utilities given in the matrices. The utility of $c_{1}$ is 10 and $c_{2}$ is 5 , i.e, $c_{1}$ is the dominant action for $c$.

can change the radius and coradius of $\mathrm{s}_{-h}$. Therefore, for $\mathrm{s}$ to be robust with respect to player $h$, the condition

$$
R_{d}\left(\mathrm{~s}^{\prime}\right)>\mathrm{CR}\left(\mathrm{s}^{\prime}\right)
$$

must be satisfied for all $\mathrm{s}^{\prime} \in L^{\mathrm{cnf}}(\mathrm{s}, h)$.

We illustrate the implications of the result in Prop. 1 through an example. Consider a game with three players $N_{p}=\{a, b, c\}$. In the standard setup, players $a$ and $b$ have identical interests, i.e., their utilities are identical for all action profiles. For player $c$, action $c_{1}$ strictly dominates $c_{2}$. Thus, the game has two pure NE in the standard setup, which are $\left(a_{1}, b_{1}, c_{1}\right)$ and $\left(a_{3}, b_{3}, c_{1}\right)$. To check for stochastically stable states, we need to compute radius and coradius for both of the NE. The basin of attraction of the NE $\left(a_{1}, b_{1}, c_{1}\right)$ contains all the states except $\left(a_{3}, b_{3}, c_{1}\right)$. The minimum resistance path from $\left(a_{1}, b_{1}, c_{1}\right)$ to $\mathrm{BA}^{c}\left(\left(a_{1}, b_{1}, c_{1}\right)\right)$ is

$$
\omega=\left(\left(a_{1}, b_{1}, c_{1}\right),\left(a_{1}, b_{3}, c_{1}\right),\left(a_{3}, b_{3}, c_{1}\right)\right) .
$$

The minimum resistance path entering $\mathrm{BA}\left(\left(a_{1}, b_{1}, c_{1}\right)\right)$ from outside is $\left(\left(a_{3}, b_{3}, c_{1}\right),\left(a_{1}, b_{3}, c_{1}\right)\right)$. Therefore,

$$
R_{d}\left(\left(a_{1}, b_{1}, c_{1}\right)\right)=3 \text { and } \operatorname{CR}\left(\left(a_{1}, b_{1}, c_{1}\right)\right)=2 .
$$

Since $R_{d}\left(\left(a_{1}, b_{1}, c_{1}\right)>\operatorname{CR}\left(\left(a_{1}, b_{1}, c_{1}\right)\right)\right.$, the state $\left(a_{1}, b_{1}, c_{1}\right)$ is stochastically stable. For $\left(a_{3}, b_{3}, c_{1}\right)$, the minimum resistance path from $\left(a_{3}, b_{3}, c_{1}\right)$ to $\operatorname{BA}^{c}\left(\left(a_{3}, b_{3}, c_{1}\right)\right)$ is $\left(\left(a_{3}, b_{3}, c_{1}\right),\left(a_{1}, b_{3}, c_{1}\right),\left(a_{1}, b_{1}, c_{1}\right)\right)$. Moreover, the path that determines the coradius of $\left(a_{3}, b_{3}, c_{1}\right) \quad$ is $\quad\left(\left(a_{1}, b_{1}, c_{1}\right),\left(a_{1}, b_{3}, c_{1}\right),\left(a_{3}, b_{3}, c_{1}\right)\right)$. Therefore,

$$
R_{d}\left(\left(a_{3}, b_{3}, c_{1}\right)\right)=2 \text { and } \operatorname{CR}\left(\left(a_{3}, b_{3}, c_{1}\right)\right)=3,
$$

which implies that the state $\left(a_{3}, b_{3}, c_{1}\right)$ is not stochastically stable.

Next, we study the impact of replacing one of the players with a confused player.

Case 1: Player $c$ is confused.

If player $c$ is confused, i.e., $h=c$, then transitions between the entries from the left matrix to the right matrix have resistance zero. To verify the robustness of the stochastically stable state $\mathrm{s}=\left(a_{1}, b_{1}, c_{1}\right)$ to the addition of $c$ as confused player, we apply the result of Prop. 1. We start with the set $L^{\mathrm{cnf}}(\mathrm{s}, c)$. The neighborhood $\mathcal{N}(\mathrm{s}, c)$ has one member only, which is $\left(a_{1}, b_{1}, c_{2}\right)$. Thus,

$$
L^{\mathrm{cnf}}(\mathrm{s}, c)=\left\{\left(a_{1}, b_{1}, c_{1}\right),\left(a_{1}, b_{1}, c_{2}\right)\right\} .
$$




\begin{tabular}{|c|c|c|c|c|c|}
\hline & \multicolumn{2}{|c|}{$c_{1}$} & & \multicolumn{2}{|c|}{$c_{2}$} \\
\hline & $b_{1}$ & $b_{2}$ & & $b_{1}$ & $b_{2}$ \\
\hline$a_{1}$ & $10,10,10$ & $6,5,3$ & $a_{1}$ & $10,2,6$ & $6,5,5$ \\
\hline$a_{2}$ & $7,5,3$ & $4,5,6$ & $a_{2}$ & $7,6,5$ & $8,8,8$ \\
\hline
\end{tabular}

Fig. 2. Matrix form representation of a three player game with $N_{p}=$ $\{a, b, c\}$. Player $a$ selects rows, player $b$ selects columns, player $c$ selects left or right matrix.

To compute the radius and coradius of $L^{\mathrm{cnf}}(\mathrm{s}, c)$, we observe that the minimum resistance path leaving the basin of attraction of $L^{\mathrm{cnf}}(\mathrm{s}, c)$ is still $\omega$ in (8). Similarly, the path that determines the coradius of $L^{\mathrm{cnf}}(\mathrm{s}, c)$ is $\left(\left(a_{3}, b_{3}, c_{1}\right),\left(a_{1}, b_{3}, c_{1}\right)\right)$. Therefore,

$$
R_{d}\left(L^{\mathrm{cnf}}(\mathrm{s}, c)\right)=3 \text { and } \mathrm{CR}\left(L^{\mathrm{cnf}}(\mathrm{s}, c)\right)=2 .
$$

Since $R_{d}\left(L^{\mathrm{cnf}}(\mathrm{s}, c)\right)>\mathrm{CR}\left(L^{\mathrm{cnf}}(\mathrm{s}, c)\right)$, the set $L^{\mathrm{cnf}}(\mathrm{s}, c)$ is stochastically stable and the action profile $\mathrm{s}$ is robust to the replacement of player $c$ with a confused player.

Case 2: Player b is confused.

If player $b$ is confused, then

$$
L^{\mathrm{cnf}}(\mathrm{s}, b)=\left\{\left(a_{1}, b_{1}, c_{1}\right),\left(a_{1}, b_{2}, c_{1}\right),\left(a_{1}, b_{3}, c_{1}\right)\right\} .
$$

In the case when player $b$ randomly switches between his actions, there exist zero cost paths between any two states in the left matrix. Thus, in the long run, player $c$ will still be playing action $c_{1}$ with high probability. However, the behavior of player $a$ will be different from the standard setup. Thus, the action profile $\mathrm{s}$ is not robust to player heterogeneity if player $b$ is replaced with a confused player.

Proposition 2: Let a $\in \mathcal{A}$ be an action profile that is not stochastically stable under the standard setup. There can exist a player $h$ such that replacing it with a confused player can make $L^{\mathrm{cnf}}(\mathrm{s}, h)$ stochastically stable.

Proof: Since a is not stochastically stable, we know that $R_{d}(\mathrm{a}) \leq \mathrm{CR}(\mathrm{a})$. By replacing player $h$ with a confused player, it is straightforward to show that $R_{d}\left(L^{\mathrm{cnf}}(\mathrm{s}, h)\right)$ in the heterogeneous setup cannot be greater that $R_{d}(\mathrm{a})$ under the standard setup. Therefore, for $L^{\mathrm{cnf}}(\mathrm{s}, h)$ to be stochastically stable, we need to show that it is possible to reduce the coradius such that the radius-coradius condition for stochastic stability is satisfied. We verify our claim through a simple example.

Consider a three player game with each player having two actions. The matrix form representation of the game is given in Fig. 2. The game has two NE, which are $\mathrm{a}_{1}^{*}=\left(a_{1}, b_{1}, c_{1}\right)$ and $\mathrm{a}_{2}^{*}=\left(a_{2}, b_{2}, c_{2}\right)$. We assume that the players are updating their actions according to LLL. The basin of attraction of $\mathrm{a}_{1}^{*}$ includes all the action profiles except $\left(a_{2}, b_{2}, c_{2}\right)$ and $\left(a_{1}, b_{2}, c_{2}\right)$. The paths that determine the $R_{d}\left(\mathrm{a}_{1}^{*}\right)$ and $\mathrm{CR}\left(\mathrm{a}_{1}^{*}\right)$ are

$$
\begin{aligned}
& \omega_{\mathrm{a}_{1}^{*}, \mathrm{a}_{2}^{*}}=\left(\left(a_{1}, b_{1}, c_{1}\right),\left(a_{2}, b_{1}, c_{1}\right),\left(a_{2}, b_{1}, c_{2}\right),\left(a_{2}, b_{2}, c_{2}\right)\right), \\
& \omega_{\mathrm{a}_{2}^{*}, \mathrm{a}_{1}^{*}}=\left(\left(a_{2}, b_{2}, c_{2}\right),\left(a_{2}, b_{1}, c_{2}\right),\left(a_{1}, b_{1}, c_{2}\right),\left(a_{1}, b_{1}, c_{1}\right)\right) .
\end{aligned}
$$

Then, the radius and coradius of this NE are $R_{d}\left(\mathrm{a}_{1}^{*}\right)=$ $R\left(\omega_{\mathrm{a}_{1}^{*}, \mathrm{a}_{2}^{*}}\right)=3$ and $\mathrm{CR}\left(\mathrm{a}_{1}^{*}\right)=R\left(\omega_{\mathrm{a}_{2}^{*}, \mathrm{a}_{1}^{*}}\right)=2$, which implies that $\mathrm{a}_{1}^{*}$ is stochastically stable. For the second NE, i.e., $\mathrm{a}_{2}^{*}=\left(a_{2}, b_{2}, c_{2}\right), R_{d}\left(\mathrm{a}_{2}^{*}\right)=R\left(\omega_{\mathrm{a}_{2}^{*}, \mathrm{a}_{1}^{*}}\right)=2$ and $\mathrm{CR}\left(\mathrm{a}_{2}^{*}\right)=R\left(\omega_{\mathrm{a}_{1}^{*}, \mathrm{a}_{2}^{*}}\right)=3$. Thus, $\mathrm{a}_{2}^{*}$ is not stochastically stable.

Now suppose that player $a$ is replaced with a confused player, i.e., $h=a$. Then

$$
L^{\mathrm{cnf}}\left(\mathrm{a}_{1}^{*}, a\right)=\left\{\left(a_{1}, b_{1}, c_{1}\right),\left(a_{2}, b_{1}, c_{1}\right)\right\}
$$

The radius of $\mathrm{a}_{1}^{*}$ under the heterogeneous setup is $R_{d}\left(\mathrm{a}_{1}^{*}\right)=$ 0 , because the transition from $\left(a_{1}, b_{1}, c_{1}\right)$ to $\left(a_{2}, b_{1}, c_{1}\right)$ now has zero resistance and the transition from $\left(a_{2}, b_{1}, c_{1}\right)$ to $\left(a_{2}, b_{1}, c_{2}\right)$ already had zero resistance. Therefore, the action profile $a_{1}^{*}$, which was stochastically stable under the standard setup, is not robust to the randomness introduced by player a.

$$
\begin{aligned}
& \text { For the other } \mathrm{NE} \mathrm{a}_{2}^{*}=\left(a_{2}, b_{2}, c_{2}\right) \\
& \qquad L^{\mathrm{cnf}}\left(\mathrm{a}_{2}^{*}, a\right)=\left\{\left(a_{2}, b_{2}, c_{2}\right),\left(a_{1}, b_{2}, c_{2}\right)\right\}
\end{aligned}
$$

The radius and coradius of $L^{\mathrm{cnf}}\left(\mathrm{a}_{2}^{*}, a\right)$ under the heterogeneous setup are are as follows:

$$
R_{d}\left(L^{\mathrm{cnf}}\left(\mathrm{a}_{2}^{*}, a\right)\right)=2, \operatorname{CR}\left(L^{\mathrm{cnf}}\left(\mathrm{a}_{2}^{*}, a\right)\right)=0,
$$

which implies that $L^{\mathrm{cnf}}\left(\mathrm{a}_{2}^{*}, a\right)$ will be stochastically stable if player $a$ is replaced with a confused player, which proves the proposition statement.

The main takeaway from this section is that the presence of even a single confused player can significantly alter the long-term behavior of the entire population.

\section{Stubborn Player: No Action Updates}

A stubborn player is one who selects one action from his action set in the beginning and then sticks with it, i.e., he never updates his action no matter how many revision opportunities he gets. Consequently, having a stubborn player restricts the state space over which the Markov chain induced by a stochastic learning dynamics evolves. Let $\mathcal{A}$ be the set of joint action profiles in the standard setup. Replacing player $h$ with a stubborn player restricts $\mathcal{A}$ to $\mathcal{A}^{\text {stb }}(h)$, where

$$
\mathcal{A}^{\mathrm{stb}}(h)=\left\{\mathrm{a} \in \mathcal{A} \mid \mathrm{a}=\left(a^{\mathrm{stb}}, \mathrm{a}_{-h}\right) \text { for all } \mathrm{a}_{-h} \in \mathcal{A}_{-h}\right\} .
$$

Here $a^{\text {stb }}$ is the action that the stubborn player will always play. By replacing $h$ with a stubborn player that only plays $a^{\text {stb }}$, the resistance between action profiles is updated as follows:

$$
R^{\mathrm{stb}}\left(\mathrm{a}, \mathrm{a}^{\prime}\right)=\left\{\begin{array}{cc}
R\left(\mathrm{a}, \mathrm{a}^{\prime}\right) & \text { if } a_{h}=a_{h}^{\prime}=a^{\mathrm{stb}} \\
\infty & \text { Otherwise }
\end{array}\right.
$$

Proposition 3: Let $\mathrm{s}=\left(s_{h}, \mathrm{~s}_{-h}\right)$ be a stochastically stable action profile under the standard setup for a stochastic learning dynamics. There can exist scenarios in which $\mathrm{s}$ will not be robust to the replacement of some player $h$ with a stubborn player. Even if $\mathrm{s}_{h}=a_{\mathrm{stb}}$, the action profile $\mathrm{s}$ may not be stochastically stable under the heterogeneous setup with a stubborn player.

Proof: To prove the statement, we present two conditions in which $\mathrm{s}$ will not be robust to the addition of a stubborn player, i.e., we will provide sufficient conditions 
for $\left(a^{\text {stb }}, \mathrm{s}_{-h}\right)$ to not be stochastically stable.

Condition 1: If $s_{h} \neq a^{\text {stb }}$ and there exists an action profile $\mathrm{a}=\left(a^{\mathrm{stb}}, \mathrm{a}_{-h}\right)$ such that the Hamming distance $d_{H}\left(\mathrm{~s}_{-h}, \mathrm{a}_{-h}\right)=1, R\left(\left(a^{\mathrm{stb}}, \mathrm{s}_{-h}\right),\left(a^{\mathrm{stb}}, \mathrm{a}_{-h}\right)\right)=0$ and $R^{\Omega}\left(\left(a^{\mathrm{stb}}, \mathrm{a}_{-}\right),\left(a^{\mathrm{stb}}, \mathrm{s}_{-h}\right)\right)>0$, then $\left(a^{\mathrm{stb}}, \mathrm{s}_{-h}\right)$ is not stochastically stable. If this condition is satisfied then $\left(a^{\text {stb }}, \mathrm{s}_{-h}\right)$ cannot be stochastically stable because there will be a zero resistance path from $\left(a^{\mathrm{stb}}, \mathrm{s}_{-h}\right)$ to $\mathrm{a}^{\prime}$ whereas all the paths from $\mathrm{a}^{\prime}$ to $\left(a^{\mathrm{stb}}, \mathrm{s}_{-h}\right)$ will have non-zero resistance.

Condition 2: Let $s_{h}=a^{\text {stb }}$, i.e., $\left(a^{\text {stb }}, \mathrm{s}_{-h}\right)$ is stochastically stable in the standard setup. Even in this case, it is not guaranteed that $\left(a^{\text {stb }}, \mathrm{s}_{-h}\right)$ will be stochastically stable under the heterogeneous setup. For the standard setup, we know that if $s_{h}=a^{\text {stb }}$, then $R_{d}\left(\left(a^{\text {stb }}, \mathrm{s}_{-h}\right)\right)>\operatorname{CR}\left(\left(a^{\text {stb }}, \mathrm{s}_{-h}\right)\right)$. Let

$$
H=R_{d}\left(\left(a^{\mathrm{stb}}, \mathrm{s}_{-h}\right)\right)-\mathrm{CR}\left(\left(a^{\mathrm{stb}}, \mathrm{s}_{-h}\right)\right) .
$$

However, the resistance function $R^{\text {stb }}$ implies that the number of paths starting from $\mathrm{s}$ and leaving the basin of attraction of $\left(a^{\text {stb }}, \mathrm{s}_{-h}\right)$ is potentially reduced because any path in which player $h$ needs to change his action will have infinite resistance. Let $\omega_{\min }$ be the least resistance path from $\mathrm{s}$ to $\mathrm{BA}^{c}\left(a^{\text {stb }}, \mathrm{s}_{-}\right)$under the standard setup. If $\omega_{\min }$ involves an action profile with $a_{h} \neq a^{\text {stb }}$, that path will have infinite resistance in the heterogeneous setup. Any path other than $\omega_{\text {min }}$ will have resistance higher than $R\left(\omega_{\text {min }}\right)$. Therefore, it is guaranteed that in the heterogeneous setup, the radius $R_{d}\left(\left(a^{\mathrm{stb}}, \mathrm{s}_{-}-h\right)\right)$ will either increase or remain the same as under the standard setup. We define $R_{d}^{\mathrm{stb}}(\mathrm{s})$ as the difference in radius of $\left(a^{\mathrm{stb}}, \mathrm{s}_{-h}\right)$ under the heterogeneous and the standard setup.

For the coradius of $\left(a^{\text {stb }}, \mathrm{s}_{-}\right)$, similar arguments are valid. Let $\omega$ be the path that determines the coradius of $\left(a^{\text {stb }}, \mathrm{s}_{-h}\right)$. If it involves any $a_{h}$ other than $a^{\text {stb }}$, the resistance of the path will be infinite. Consequently, the coradius will also increase. Let $\mathrm{CR}^{\mathrm{stb}}(\mathrm{s})$ be the difference in coradius between the heterogeneous and the standard setup. If

$$
\mathrm{CR}^{\mathrm{stb}}(\mathrm{s})-R_{d}^{\mathrm{stb}}(\mathrm{s})>H
$$

the action profile $\left(a^{\text {stb }}, \mathrm{s}_{-} h\right)$ will not be stochastically stable in the heterogeneous case.

Conditions 1 and 2 are not the only conditions under which a stochastically stable profile is not robust to the addition of a stubborn player. However, these conditions establish the fact that a stochastically stable action profile $\mathrm{s}$ in the standard setup may not be robust to the addition of a stubborn player $h$ even if $s_{h}=a^{\text {stb }}$.

\section{CONCLUSIONS}

We considered a class of stochastic learning dynamics in games for a heterogeneous population of players in which one of the players had a different rule for updating his actions. To analyze the impact of the heterogeneous player, we defined a new notion of robustness to player heterogeneity. The analysis and results presented in this work open up a new direction of research in the well-studied area of stochastic learning dynamics. Some interesting open problems related to player heterogeneity are as follows:

1) One important problem is to generalize this analysis when we have multiple heterogeneous players of different types in the population.

2) An exciting research problem is to compare different stochastic learning dynamics like Log-Linear Learning, Adaptive Play, and Metropolis Learning based on the proposed criterion of robustness to player heterogeneity.

3) Another interesting problem is to analyze the impact of underlying game structure and interaction network topology on robustness to player heterogeneity.

4) We want to highlight here that stochastic learning dynamics like Log-Linear Learning also have applications in various engineering applications like resource allocation problems, sensor networks, and self reconfigurable systems as well (see e.g., [5], [12], and [13]). If we consider the example of sensor network coverage problem, a confused or a stubborn behavior of a sensor will imply that the sensor is malfunctioning. Thus, an interesting research direction is to formalize the proposed notion of robustness for designing engineering systems like sensor networks that are robust to device failures and strategic attacks.

\section{REFERENCES}

[1] H. P. Young, "The evolution of conventions," Econometrica, vol. 61, no. 1, pp. 57-84, January 1993.

[2] M. Kandori and R. Rob, "Evolution of equilibria in the long run: A general theory and applications," Journal of Economic Theory, vol. 65, no. 2, pp. 383-414, 1995.

[3] L. E. Blume, "The statistical mechanics of strategic interaction," Games and economic behavior, vol. 5, no. 3, pp. 387-424, 1993.

[4] C. Alós-Ferrer and N. Netzer, "The logit-response dynamics," Games and Economic Behavior, vol. 68, no. 2, pp. 413-427, 2010.

[5] H. Jaleel and J. S. Shamma, "Transient response analysis of metropolis learning in games," IFAC-PapersOnLine, vol. 50, no. 1, pp. 96619667, 2017

[6] J. R. Marden and J. S. Shamma, "Revisiting log-linear learning: Asynchrony, completeness and payoff-based implementation," Games and Economic Behavior, vol. 75, no. 2, pp. 788-808, 2012.

[7] E. Yildiz, D. Acemoglu, A. E. Ozdaglar, A. Saberi, and A. Scaglione, "Discrete opinion dynamics with stubborn agents," Available at SSRN 1744113, 2011.

[8] S. Morris, "Contagion," The Review of Economic Studies, vol. 67, no. 1 , pp. $57-78,2000$.

[9] S. Sundaram and B. Gharesifard, "Distributed optimization under adversarial nodes," IEEE Transactions on Automatic Control, 2018.

[10] W. Abbas, A. Laszka, and X. Koutsoukos, "Improving network connectivity and robustness using trusted nodes with application to resilient consensus," IEEE Transactions on Control of Network Systems, vol. 5, no. 4, pp. 2036-2048, 2018.

[11] G. Ellison, "Basins of attraction, long-run stochastic stability, and the speed of step-by-step evolution," The Review of Economic Studies, vol. 67 , no. 1 , pp. $17-45,2000$.

[12] J. R. Marden and J. S. Shamma, "Game theory and distributed control," Handbook of game theory, vol. 4, pp. 861-900, 2012.

[13] J. R. Marden and A. Wierman, "Distributed welfare games with applications to sensor coverage," in Decision and Control, 2008. CDC 2008. 47th IEEE Conference on. IEEE, 2008, pp. 1708-1713. 